\title{
Comparison of Average Inhibition Zones of Methanolic Extracts of Chaetomium globosum on the Growth of Different Groups of Pathogenic Micro-Organisms
}

\author{
Haider Mashkoor Hussein', Sanaa Hasan Abdalakhwa², Raghda A. Razaq ${ }^{3}$ \\ ${ }^{1}$ Assist. Prof., Department of Environment, College of Science, University of Al-Qadisiyah, Iraq, ${ }^{2}$ Lecturer, College \\ of Basic Education, University of Babylon, Hillah City, Iraq, ${ }^{3}$ Bachelor, Department of Biology, College of Science \\ for Women, University of Babylon, Iraq
}

\begin{abstract}
The objectives of this study were analysis of the secondary metabolite products and evaluation of antibacterial activity. The FTIR analysis of Chaetomium globosum proved the presence of functional group assignment Alkyl halides, Amide, and Alkane with Peak (Wave number cm- ${ }^{1}$ ) 1018.41 (Strong), 1238.30 (Strong), 1317.38 (Strong), 1379.10 (Strong), 1614.42 (Bending), 2850.79 (Strong), 2920.23 (Strong). Zone of inhibition (mm) of test bacterial strains to Chaetomium globosum bioactive compounds and standard antibiotics were $(4.00 \pm 0.31),(4.77 \pm 0.13),(5.00 \pm 0.16),(3.22 \pm 0.41)$, and $(4.29 \pm 0.11)$ uses Chaetomium globosum bioactive compounds, and (2.01 \pm 0.10$),(1.77 \pm 0.41),(1.98 \pm 0.11),(2.00 \pm 0.30)$, and $(1.05 \pm 0.42)$ uses Rifambin, and (2.42 \pm 0.18$),(2.12 \pm 0.46),(1.40 \pm 0.12),(1.00 \pm 0.10)$, and (2.90 \pm 0.47$)$ uses Kanamycin for Staphylococcus aureus, Escherichia coli, Proteus mirabilis, Klebsiella pneumonia, Pseudomonas eurogenosa respectively. Chaetomium globosum was very highly active against Escherichia coli (4.77 \pm 0.13$)$.
\end{abstract}

Keywords: Chaetomium globosum, FT-IR, Secondary metabolites.

\section{Introduction}

Chaetomium species are widespread worldwide in soil and plant debris. Chaetomium globosum is a well-known mesophilic member of the mold family Chaetomiaceae. It is a saprophytic fungus that primarily resides on plants ${ }^{1}$, soil, straw, and dung. Both the C. globosum hyphae and the spores contain antigens such as $\mathrm{Chg} 45$, to induce $\operatorname{IgE}$ and $\operatorname{IgG}$ antibody production in allergic individuals. Although the $\operatorname{IgE}$ upsurge is transient, increased IgG levels persist in the serum. This can lead to non-atopic asthma, sinusitis, and respiratory illnesses in the residents of contaminated buildings. ${ }^{2}$ Such allergic onsets can be prevented with the use of potassium chlorate in building materials. Chlorate, toxic to many fungal strains, disrupts nitrate reduction in fungi by using fungal nitrate reductase to produce the toxic chlorite. Although it is unclear as to whether $\mathrm{C}$. globosum contains nitrate reductase, chlorate is still a well known C. globosum toxin. However, even though chlorate suppresses perithecia formation, it does not affect hyphal growth nor sporulation. Secondary metabolites often play an important role in plant defense against herbivory and other interspecies defenses. Humans use secondary metabolites as medicines, flavourings, pigments, and recreational drugs ${ }^{4}$. Secondary metabolites aid a host in important functions such as protection, competition, and species interactions, but are not necessary for survival. One important defining quality of secondary metabolites is their specificity. Usually, secondary metabolites are specific to an individual species, though there is considerable evidence that horizontal transfer across species or genera of entire pathways plays an important role in bacterial (and, likely, fungal) evolution. Research also shows that secondary metabolism can affect different species in varying ways. In the same forest, four separate species of arboreal marsupial folivores reacted differently to a secondary metabolite in eucalypts. ${ }^{6}$

\section{Materials and Method}

After growing on potato dextrose agar (PDA) medium at $25^{\circ} \mathrm{C}$ for 4 days, the fresh mycelium of strain C. globosum No. 04 was inoculated in liquid medium 
containing: Oat flour $80 \mathrm{~g}$, maltose $10 \mathrm{~g}$, yeast extracts $2 \mathrm{~g}$ dissolved in $1,000 \mathrm{~mL} \mathrm{dH} 2 \mathrm{O}$. The $\mathrm{pH}$ was adjusted to 6.0 before autoclaving. Fermentation was carried out in $2 \mathrm{~L}$ flasks each containing $1 \mathrm{~L}$ medium on a rotary shaker at $180 \mathrm{r} / \mathrm{min}, 25^{\circ} \mathrm{C}$ for 8 days, and the cultures were used for the extraction and isolation.

Fourier transform infrared spectrophotometer (FTIR): The powdered sample of Chaetomium globosum products was treated for FTIR spectroscopy (Shimadzu, IR Affinity, Japan). The sample was run at infrared region between $400 \mathrm{~nm}$ and $4000 \mathrm{~nm}$.

Antibacterial activity: Determination of antibacterial activities of pure extract of C. globosum cultures performed using streak-plate method. Mueller Hinton agar plates were prepared and inoculated with $C$. globosum cultures by a single streak of inoculum in the center of the petri dish and incubated at $27^{\circ} \mathrm{C}$ for 4 days. Antagonism was measured by the determination of the size of the inhibition zone in millimeters ${ }^{7}$.

\section{Results and Discussion}

The FTIR analysis of Chaetomium globosum proved the presence of functional group assignment Alkyl halides, Amide, and Alkane with Peak (Wave number $\mathrm{cm}^{-1}$ ) 1018.41 (Strong), 1238.30 (Strong), 1317.38 (Strong), 1379.10 (Strong), 1614.42 (Bending), 2850.79 (Strong), 2920.23 (Strong). Zone of inhibition (mm) of test bacterial strains to Chaetomium globosum bioactive compounds and standard antibiotics were (4.00 \pm 0.31$), \quad(4.77 \pm 0.13), \quad(5.00 \pm 0.16), \quad(3.22 \pm 0.41)$, and $(4.29 \pm 0.11)$ uses Chaetomium globosum bioactive compounds, and (2.01 \pm 0.10$),(1.77 \pm 0.41),(1.98 \pm 0.11)$, $(2.00 \pm 0.30)$, and $(1.05 \pm 0.42)$ uses Rifambin, and (2.42 \pm 0.18$), \quad(2.12 \pm 0.46), \quad(1.40 \pm 0.12), \quad(1.00 \pm 0.10)$, and (2.90 \pm 0.47$)$ uses Kanamycin for Staphylococcus aureus, Escherichia coli, Proteus mirabilis, Klebsiella pneumonia, Pseudomonas eurogenosa respectively. Chaetomium globosum was very highly active against Escherichia coli (4.77 \pm 0.13$)$. Infrared spectroscopy provides a useful method for herbal analysis and elucidate the compounds structures as well as for quantitative analysis of drugs. Recently, a number of plants have been reported for antibacterial properties across the world ${ }^{19}$. It is hoped that this study would direct to the establishment of some compounds that could be used to invent new and more potent antibacterial drugs of natural origin. Further work will emphasize the isolation and characterization of active principles responsible for bio-efficacy and bioactivity. The differences in the susceptibilities of Gram positive and Gram negative bacteria to Streptomyces extracts have been observed by previous workers ${ }^{13}$. Gram negative bacteria are inherently more resistant to antimicrobials than Gram positive organisms and this has been ascribed to the combined exclusion of antimicrobial compounds by double membrane barrier and transmembrance efflux present in this group of organisms. The three main classes of fungal secondary metabolites are: polyketides, nonribosomal peptides and terpenes. Although fungal SMs are not required for growth they play an essential role in survival of fungi in their ecological niche ${ }^{11}$. The goal of absorption spectroscopy techniques (FTIR, ultraviolet-visible ("UV-Vis") spectroscopy, etc.) is to measure how much light a sample absorbs at each wavelength. The most straightforward way to do this, the "dispersive spectroscopy" technique, is to shine a monochromatic light beam at a sample, measure how much of the light is absorbed, and repeat for each different wavelength. (This is how some UV-vis spectrometers work, for example).

Table 1. FT-IR peak values of solid analysis of Chaetomium globosum

\begin{tabular}{|c|c|c|c|c|c|c|c|c|}
\hline No. & $\begin{array}{c}\text { Peak (Wave } \\
\text { number cm- })\end{array}$ & Intensity & $\begin{array}{c}\text { Corr. } \\
\text { Area }\end{array}$ & $\begin{array}{c}\text { Type of } \\
\text { Intensity }\end{array}$ & Bond & $\begin{array}{c}\text { Type of } \\
\text { Vibration }\end{array}$ & $\begin{array}{c}\text { Functional group } \\
\text { assignment }\end{array}$ & $\begin{array}{c}\text { Group } \\
\text { frequency }\end{array}$ \\
\hline 1. & 1018.41 & 61.727 & 0.277 & Strong & C-F & Stretch & alkyl halides & $1000-1400$ \\
\hline 2. & 1238.30 & 81.739 & 0.396 & Strong & C-F & Stretch & alkyl halides & $1000-1400$ \\
\hline 3. & 1317.38 & 83.193 & 0.054 & Strong & C-F & Stretch & alkyl halides & $1000-1400$ \\
\hline 4. & 1379.10 & 81.889 & 0.035 & Strong & C-F & Stretch & alkyl halides & $1000-1400$ \\
\hline 5. & 1614.42 & 79.693 & 0.163 & Bending & N-H & Stretch & Amide & $1550-1640$ \\
\hline 6. & 2850.79 & 86.184 & 0.321 & Strong & C-H & Stretch & Alkane & $2850-3000$ \\
\hline 7. & 2920.23 & 81.949 & 0.993 & Strong & C-H & Stretch & Alkane & $2850-3000$ \\
\hline
\end{tabular}




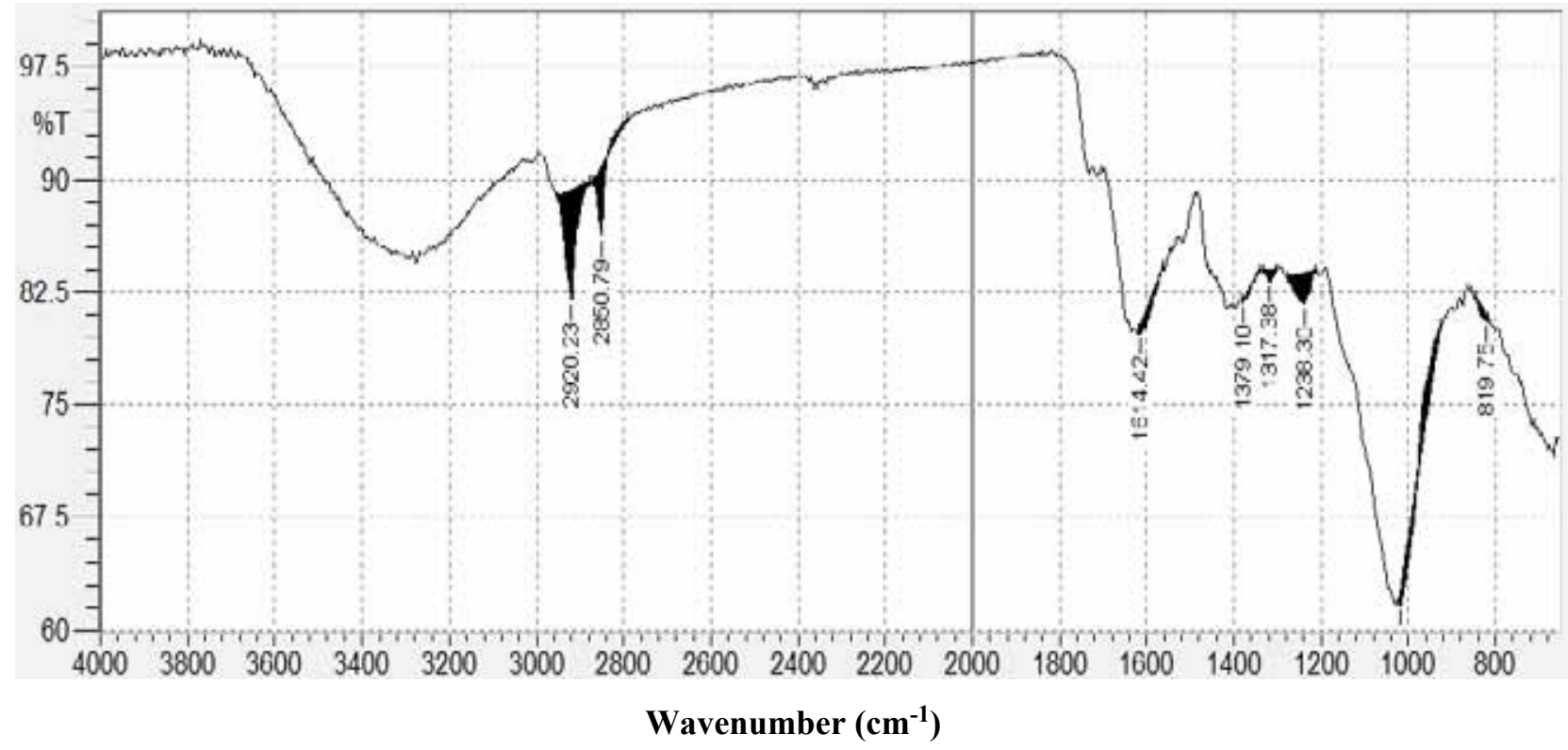

Figure 1. Fourier-transform infrared spectroscopic profile solid analysis of Chaetomium globosum

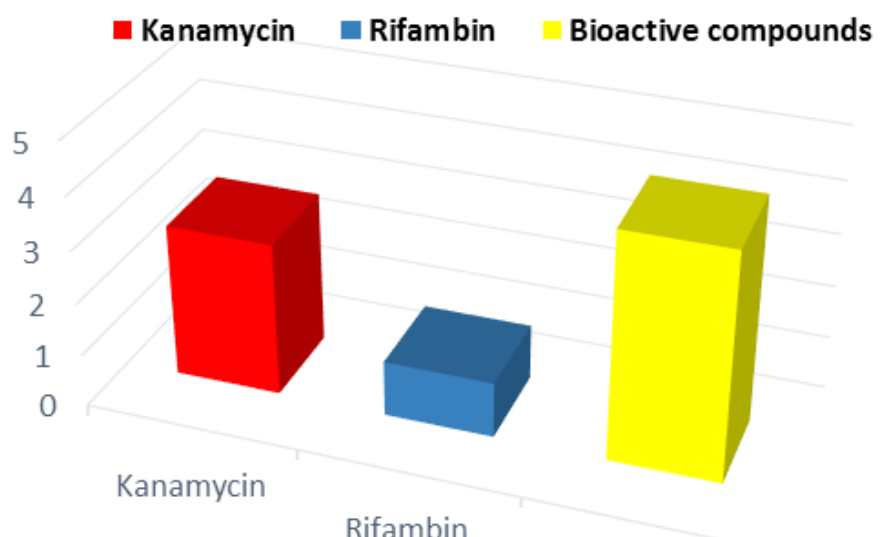

Bioactive

compounds

Figure 2. Anti-Bacterial activity against Pseudomonas eurogenosa

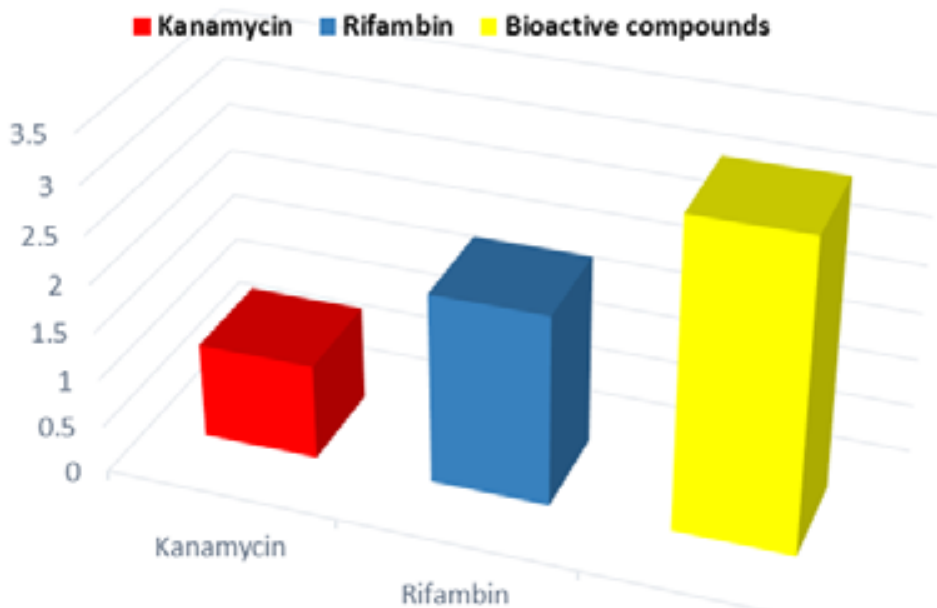

Bioactive

compounds

Figure 3. Anti-Bacterial activity against Klebsiella pneumonia 


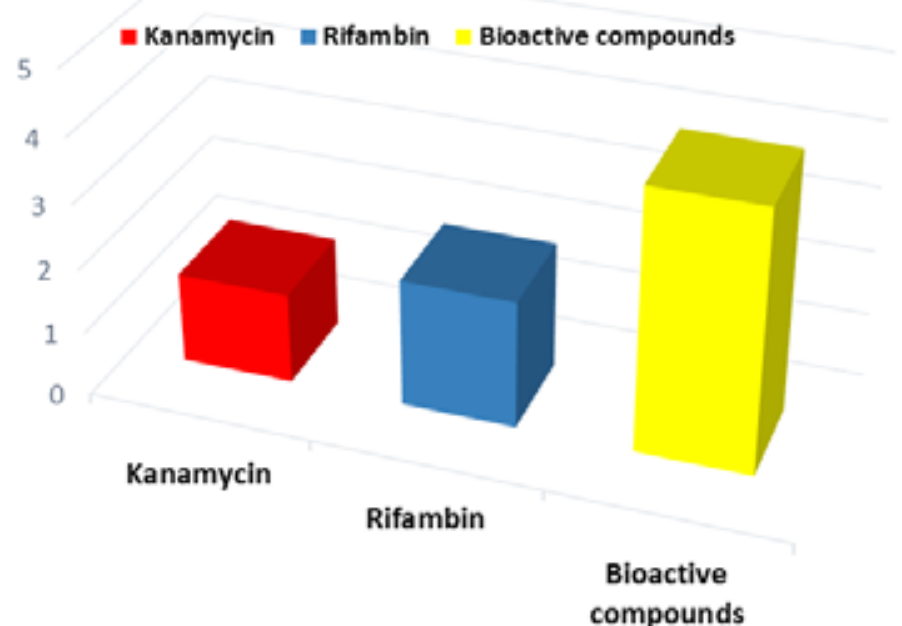

Figure 4. Anti-Bacterial activity against Proteus mirabills

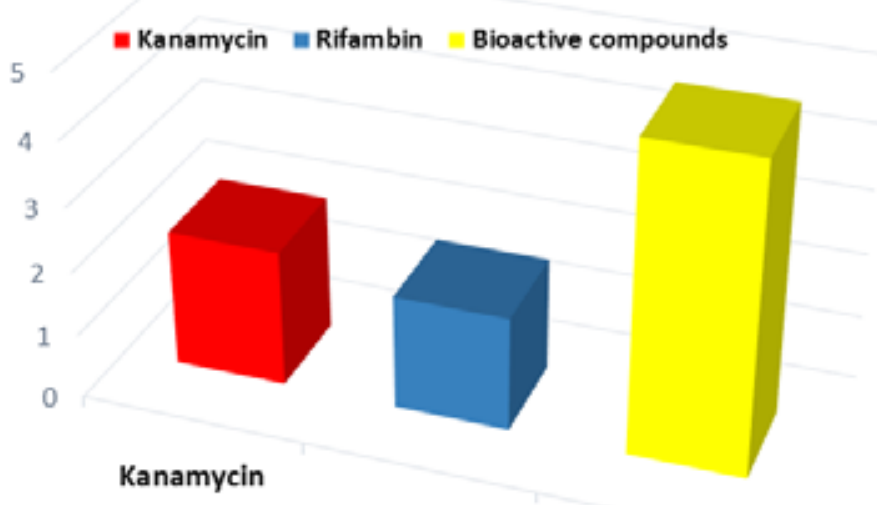

Rifambin

Bioactive

compounds

Figure 5. Anti-Bacterial activity against Escherichia colf

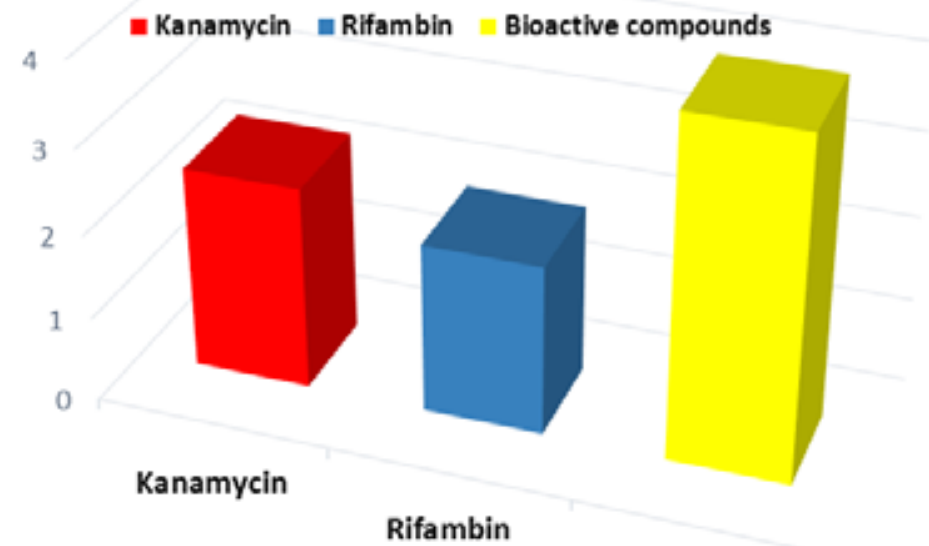

Bioactive

compounds

Figure 6. Anti-Bacterial activity against Staphylococcus aureus 


\section{Conclusion}

Chaetomium globosum proved the presence of functional group assignment Alkyl halides, Amide, and Alkane. Chaetomium globosum bioactive compounds and standard antibiotics were $(4.00 \pm 0.31),(4.77 \pm 0.13)$,

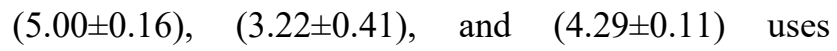
Chaetomium globosum bioactive compounds, and (2.01 \pm 0.10$), \quad(1.77 \pm 0.41), \quad(1.98 \pm 0.11), \quad(2.00 \pm 0.30)$, and $(1.05 \pm 0.42)$ uses Rifambin, and $(2.42 \pm 0.18)$, (2.12 \pm 0.46$),(1.40 \pm 0.12),(1.00 \pm 0.10)$, and $(2.90 \pm 0.47)$ uses Kanamycin for Staphylococcus aureus, Escherichia coli, Proteus mirabilis, Klebsiella pneumonia, Pseudomonas eurogenosa respectively. Chaetomium globosum was very highly active against Escherichia coli $(4.77 \pm 0.13)$.

Financial Disclosure: There is no financial disclosure.

Conflict of Interest: None to declare.

Ethical Clearance: In our research, all protocols were approved under the Department of Biology, College of Science for women, University of Babylon, Hillah city, Iraq and all method were carried out in accordance with approved guidelines.

\section{References}

1. Domsch K, Gams W, Anderson T. Compendium of Soil Fungi" London, New York: Academic Press. 1980.

2. Provost N, Shi C, She Y, Cyr T, Miller, D. Characterization of an antigenic chitosanase from the cellulolytic fungus Chaetomium globosum". Medical Mycology. 2013; 51:290-299.

3. Kim D, Lee M, Suh, M, Ha G, Kim, H, Choi J. Onychomycosis Caused by Chaetomium globosum". Ann Dermatol. 2008; 25(2): 232-236.

4. Alon T, Amirav A. Isotope Abundance Analysis Method and Software for Improved Sample Identification with the Supersonic GC-MS". Rapid Communications in Mass Spectrometry. 2006; 20 (17): 2579-2588.

5. Skarysz A. Convolutional neural networks for automated targeted analysis of raw gas chromatography-mass spectrometry data". International Joint Conferences on Neural Networks (2018) Rio de Janeiro. 2018; 1-8.

6. Hübschmann H. Handbook of GC-MS : Fundamentals and Applications (3 ed.). John Wiley
\& Sons, Incorporated. 2015; 735.

7. Tsivou M, Kioukia-Fougia, N. An overview of the doping control analysis during the Olympic Games of 2004 in Athens, Greece". Analytica Chimica Acta. 2006; 555: 1-13.

8. Ghooshkhaneh, N, Golzarian M, Mamarabadi M. Detection and classifcation of citrus green mold caused by Penicillium digitatum using multispectral imaging. J Sci Food Agric. 2018; 98(9): 3542-3550

9. United States Department of Agriculture - Foreign Agricultural Service, 2019. Citrus: World Markets and Trade, https://apps.fas. usda.gov/psdonline/ circulars/citrus.2019.

10. Macarisin D. Penicillium digitatum suppresses production of hydrogen peroxide in host tissue during infection of citrus fruit. Phytopathology. 2007; 97: 1491-1500.

11. Hao W, Li H, Hu M, Yang, L, Rizwan-ul-Haq M. Integrated control of citrus green and blue mold and sour rot by Bacillus amyloliquefaciens in combination with tea saponin. Postharvest Biol Technol. 2011; 59: 316-323

12. Frisvad, J, Samson, R. Polyphasic taxonomy of Penicillium subgenus Penicillium A guide to identifcation of food and airborne terverticillate Penicillia and their mycotoxins. Stud Mycol. 2004; 49: $1-174$.

13. Kanetis L, Förster H, Adaskaveg J. Determination of natural resistance frequencies in Penicillium digitatum using a new airsampling method and characterization of Fludioxonil- and PyrimethanilResistant isolates. Phytopathology. 2010; 100: 738743

14. Strano M, Altieri G, Admane, N. Advance in Citrus Postharvest Management: Diseases, Cold Storage and Quality Evaluation. In: Gill, H. \& Garg, H. Citrus pathology. 2017; Ch 7,139-159

15. Azzollini A. Dynamics of Metabolite Induction in Fungal Co-cultures by Metabolomics at Both Volatile and Non-volatile Levels. Front Microbiol. 2018; 9

16. Demain, AL. Importance of microbial natural products and the need to revitalize their discovery. J Ind Microbiol Biotechnol. 2014; 41: 185-201

17. Bode H, Bethe B, Höfs R, Zeeck A. Big efects from small changes: Possible ways to explore nature's chemical diversity. Chem Bio Chem. 2002; 3: 619-627. 
18. Pan R, Bai X, Chen J, Zhang, H, Wang H. Exploring Structural Diversity of Microbe Secondary Metabolites Using OSMAC Strategy: A Literature Review. Front Microbiol. 2019; 10: 294

19. Keller NP. Fungal secondary metabolites: regulation, function and drug discovery. Nat Rev Microbiol. 2019; 17:167-180.
20. Aghcheh, R. K. \& Kubicek, C. P. Epigenetics as an emerging tool for improvement of fungal strains used in biotechnology. Appl Microbiol Biotechnol. 2015; 99(15): 6167-6181.

21. Madigan MT, Martiko JM, Parker J. Antibiotics: Isolation and characterization, in: Brook Biology of Microorganisms, 8th edn. Prentice-Hall International Inc. New Jersey. 1997; 440-442. 